Research Paper

\title{
A high triglyceride-glucose index is associated with left ventricular dysfunction and atherosclerosis
}

\author{
Tai-Hua Chiu'1\#, Hui-Ju Tsai2,3,4\#, Hsin-Ying Clair Chiou' ${ }^{5}$, Pei-Yu Wu 6,7, Jiun-Chi Huang6,7,8, Szu-Chia \\ Chen ${ }^{4,6,7,8} \bowtie$ \\ 1. Department of General Medicine, Kaohsiung Medical University Hospital, Kaohsiung, Taiwan \\ 2. Department of Family Medicine, Kaohsiung Municipal Ta-Tung Hospital, Kaohsiung Medical University Hospital, Kaohsiung Medical University, \\ Kaohsiung, Taiwan \\ 3. Graduate Institute of Clinical Medicine, College of Medicine, Kaohsiung Medical University, Kaohsiung, Taiwan \\ 4. Research Center for Environmental Medicine, Kaohsiung Medical University, Kaohsiung, Taiwan \\ 5. Teaching and Research Center, Kaohsiung Municipal Siaogang Hospital, Kaohsiung Medical University, Kaohsiung, Taiwan \\ 6. Division of Nephrology, Department of Internal Medicine, Kaohsiung Medical University Hospital, Kaohsiung Medical University, Kaohsiung, Taiwan \\ 7. Department of Internal Medicine, Kaohsiung Municipal Siaogang Hospital, Kaohsiung Medical University, Kaohsiung, Taiwan \\ 8. Faculty of Medicine, College of Medicine, Kaohsiung Medical University, Kaohsiung, Taiwan \\ \# These authors contributed equally to this work.
}

$\square$ Corresponding author: Szu-Chia Chen. Department of Internal Medicine, Kaohsiung Municipal Hsiao-Kang Hospital, Kaohsiung Medical University, Kaohsiung, Taiwan, 482, Shan-Ming Rd., Hsiao-Kang Dist., 812 Kaohsiung, Taiwan, R.O.C. TEL: 886- 7- 8036783 - 3441; FAX: 886- 7- 8063346; E-mail: scarchenone@yahoo.com.tw

(C) The author(s). This is an open access article distributed under the terms of the Creative Commons Attribution License (https://creativecommons.org/licenses/by/4.0/). See http://ivyspring.com/terms for full terms and conditions.

Received: 2020.09.29; Accepted: 2020.12.18; Published: 2021.01.01

\begin{abstract}
Background: The triglyceride-glucose (TyG) index has been reported to be a simple and reliable surrogate marker of insulin resistance. The aim of this study was to investigate associations between the TyG index and echocardiographic parameters including left ventricular mass (LVM), left atrial diameter (LAD) and left ventricular ejection fraction (LVEF), and markers of peripheral artery disease, ankle-brachial index ( $\mathrm{ABI}$ ) and brachial-ankle pulse wave velocity (baPWV).

Methods: A total of 823 (483 males and 340 females) patients were enrolled from 2007 to 2011 at a regional hospital in southern Taiwan. Multivariable stepwise linear regression analysis was performed to identify the factors related to echocardiographic parameters and peripheral artery disease.

Results: The patients were stratified into four groups according to TyG index quartile. Multivariable stepwise linear regression analysis showed that a higher TyG index was associated with elevated observed/predicted LVM $(p=0.081)$, increased LAD $(p=0.004)$, decreased $\operatorname{LVEF}(p=0.003)$ and lower $A B I(p=0.030)$, but not observed/predicted LVM and baPWV.

Conclusions: $A$ high TyG index was significantly associated with high LAD, low LVEF and low ABI. However, the TyG index was not significantly associated with inappropriate LVM or baPWV. The results suggest that the TyG index, as a simple indicator of insulin resistance, may reflect cardiac remodeling and dysfunction and atherosclerosis.
\end{abstract}

Key words: triglyceride-glucose index, left ventricular dysfunction, left ventricular mass, atherosclerosis

\section{Introduction}

Cardiovascular (CV) diseases account for about one-third of all deaths mortality globally, and they have become a major public health burden over the past several decades (1). Abnormal adiposity and derangement of glucose metabolism are two important components of metabolic syndrome, and they have been suggested to play major roles in the development of $\mathrm{CV}$ diseases $(2,3)$. The triglycerideglucose (TyG) index is derived from both fasting triglycerides and glucose, and it has been shown to be a novel marker of insulin resistance (IR) and to have better predictive value than traditional parameters $(4,5)$. In addition, previous studies have reported associations between the TyG index and various $\mathrm{CV}$ 
abnormalities, including hypertension (6), arterial stiffness (7) and coronary artery calcification (8). Moreover, higher TyG index levels have also been associated with a poor prognosis in patients with acute ST-elevation myocardial infarction (9), higher $\mathrm{CV}$ mortality in patients starting peritoneal dialysis (10), and future adverse CV events in patients with diabetes mellitus (DM) and acute coronary syndrome (11). Our previous study revealed an association between the TyG index and an increased risk of $\mathrm{CV}$ events in patients with type $2 \mathrm{DM}(12)$, and another study reported that a high TyG index was associated with micro- and macro-angiopathies in patients with type 2 DM (13). However, the exact mechanism underlying the association is still unclear.

Vascular screening devices have been developed to measure both ankle-brachial index (ABI) and brachial-ankle pulse wave velocity (baPWV), which have been shown to be good markers of atherosclerosis and arterial stiffness, respectively $(14,15)$. A recent study of Chinese elderly by Zhao et al. investigated associations between the TyG index and parameters of vascular damage, including $\mathrm{ABI}$, baPWV, carotid-femoral pulse wave velocity (cfPWV), carotid intima-media thickness and carotid plaque, and found a significant association between an elevated TyG index and a higher risk of nephric microvascular damage and arterial stiffness (16). However, few studies have explored associations between the TyG index and echocardiographic parameters, which can be used to evaluate cardiac function and possibly predict future CV risk $(17,18)$. Thus the aim of this study was to investigate associations between the TyG index and echocardiographic parameters including left ventricular mass (LVM), left atrial diameter (LAD) and left ventricular ejection fraction (LVEF), and markers of peripheral artery disease, $\mathrm{ABI}$ and baPWV.

\section{Materials and methods}

\section{Study Patients}

The study design has been described previously (19). In brief, we enrolled patients who underwent echocardiographic examinations from 2007 to 2011 at a regional hospital in southern Taiwan. Patients with atrial fibrillation, significant ankle edema, significant aortic or mitral valve diseases and poor image quality were excluded. A total of 823 participants (mean age $61.3 \pm 13.2$ years, 483 males and 340 females) were included in this study. The protocol was approved by the Institutional Review Board of Kaohsiung Medical University Hospital, and all enrolled patients gave written informed consent.

\section{Evaluation of Cardiac Structure and Function}

Echocardiographic examinations were performed by one experienced cardiologist using a VIVID 7 system (General Electric Medical Systems, Horten, Norway) with the participant placed in the left decubitus position breathing quietly. The cardiologist was blinded to the other data. Two-dimensional and 2-dimensionally guided standard M-mode images were obtained, and echocardiographic parameters including LAD, left ventricular posterior wall thickness in diastole (LVPWTd), left ventricular internal diameter in diastole (LVIDd), interventricular septal wall thickness in diastole (IVSTd), transmitral E wave velocity, transmitral A wave velocity, and E-wave deceleration time were measured. Left ventricular systolic function was assessed according to the LVEF. LVM was calculated using a modification of Devereux's method as follows: LVM $=1.04 \times$ ([IVSTd + LVIDd + LVPWTd] ${ }^{3}-$ LVIDd $\left.^{3}\right)$ - 13.6 g (20). Left ventricular mass index (LVMI) was calculated as LVM/body surface area. Left ventricular hypertrophy (LVH) was defined according to the 2007 European Society of Hypertension/European Society of Cardiology guidelines (21). Inappropriate LVM was defined as observed/predicted LVM. Predicted LVM was calculated as: predicted $\mathrm{LVM}=55.37+6.64 \mathrm{x}$ height $\left(\mathrm{m}^{2.7}\right)+0.64 \times$ stroke work $-18.07 \times$ sex (in which sex was coded as male $=1$ and female $=2)(22)$. Stroke work in gram-meters was calculated as systolic blood pressure $\mathrm{x}$ stroke volume $\mathrm{x} \quad 0.0144$. 'Inappropriate' LVM was defined as observed/ predicted LVM $>128 \%(22,23)$.

\section{Assessment of baPWV and ABI}

For baPWV, pulse waves were recorded in both brachial and tibial arteries to calculate the transmission time $(\Delta \mathrm{Tba})$, which was defined as the time between the initial increase in ankle and brachial waveforms $(15,24,25)$. The distance from the ankle to brachium was defined as the transmission distance and calculated according to body height. The path length from the suprasternal notch to the brachium $(\mathrm{Lb})$ was calculated as: $\mathrm{Lb}=0.2195 \mathrm{x}$ height of the patient (in $\mathrm{cm}$ ) - 2.0734. The path length from the suprasternal notch to the ankle (La) was calculated as: $\mathrm{La}=0.8129 \times$ height of the patient $($ in $\mathrm{cm})+12.328$. $\mathrm{BaPWV}$ was then calculated as: baPWV $=(\mathrm{La}-$ $\mathrm{Lb}) / \Delta \mathrm{Tba}$. The higher of bilateral baPWV values was used for analysis. ABI was measured using an ABI-form device, which measured blood pressures (BPs) in both arms and ankles using an oscillometric method, and calculated as: ankle systolic BP/arm systolic BP, with only the lower ankle systolic BP 
value being used. $A B I$ was measured once in each patient.

\section{Collection of Demographic, Medical and Laboratory Data}

Demographic and medical data including age, sex, and comorbid conditions were obtained from medical records or interviews with the patients. Body mass index (BMI) was calculated as weight/height squared $\left(\mathrm{kg} / \mathrm{m}^{2}\right)$. Laboratory data including fasting glucose, triglycerides, total cholesterol, creatinine, and hematocrit were measured from fasting blood samples using an autoanalyzer (D-68298 Mannheim COBAS Integra 400; Roche Diagnostics $\mathrm{GmbH}$, Mannheim, Germany). Blood samples were obtained within 1 month of enrollment. Serum creatinine was measured using the compensated Jaffé method on the Integra 400 system (Roche Diagnostics $\mathrm{GmbH}$ ) with calibration traceable to isotope-dilution mass spectrometry (26). Estimated glomerular filtration rate (eGFR) was calculated using the four-variable Modification of Diet in Renal Disease equation (27).

\section{Statistical Analysis}

The patients were stratified into four groups according to TyG index quartile. Data are expressed as percentages for categorical variables or mean $\pm S D$ for continuous variables. Comparisons among groups were performed using one-way analysis of variance (ANOVA) followed by a post hoc test with Bonferroni correction. Multivariate stepwise linear regression analysis was used to identify the factors associated with echocardiographic parameters (observed/ predicted LVM, LAD and LVEF) and peripheral artery disease (ABI and baPWV). Statistical analysis was performed using SPSS version 19.0 for Windows (IBM Corp, Armonk, NY). A difference was considered to be significant if the $\mathrm{P}$ value was $<0.05$.

\section{Results}

This study enrolled 823 participants stratified into four groups by TyG index quartile, with 207, 207, 206 and 203 in each group, respectively. Table 1 shows comparisons of clinical characteristics among the study groups. The cutoff values of the TyG index quartiles were $<8.4, \geq 8.4$ to $<8.8, \geq 8.8$ to $<9.3$, and $\geq$ 9.3 , respectively. The mean values \pm standard deviations of the TyG index in the four groups were $8.1 \pm 0.2,8.6 \pm 0.1,9.0 \pm 0.1$, and $9.8 \pm 0.5$, respectively. Compared to the patients in quartile 1 , those in quartile 4 were younger, and had a higher prevalence of DM, higher BMI, higher cholesterol, higher observed/predicted LVM and higher LAD.

\section{Determinants of Observed/Predicted LVM, LAD and LVEF in the Study Patients}

Table 2 summarizes the possible determinants of observed/predicted LVM, LAD and LVEF in our study patients. After adjusting for age, sex, history of DM and hypertension, mean arterial pressure, hematocrit, eGFR and total cholesterol, multivariate stepwise linear regression analysis revealed that young age $(p=0.013)$, DM $(p=0.021)$, low mean arterial pressure $(p<0.001)$ and low eGFR $(p<0.001)$ were significantly associated with high observed/predicted LVM, but that TyG index was not (unstandardized coefficient $\beta, 4.401 ; 95 \% \mathrm{CI},-0.540$ to 9.342; $p=0.081$ ). Regarding LAD, a high TyG index (unstandardized coefficient $\beta, 4.401 ; 95 \% \mathrm{CI},-0.540$ to 9.342; $p=0.081)$, male sex $(p<0.001)$, and low eGFR ( $p$ $<0.001)$ were significantly correlated with high LAD. In addition, a high TyG index (unstandardized coefficient $\beta,-2.012 ; 95 \% \mathrm{CI},-3.338$ to $-0.686 ; p=0.003$ ), young age $(p=0.026)$, male $(p=0.005)$, no history of hypertension $(p<0.001)$, coronary artery disease $(p<$ $0.001)$ and low eGFR $(p<0.001)$ were significantly correlated with low LVEF.

Table 1. Comparison of baseline characteristics according to TyG index quartiles

\begin{tabular}{|c|c|c|c|c|}
\hline Characteristics & $\begin{array}{l}\text { Quartile } 1 \\
<8.4 \\
(\mathrm{n}=207)\end{array}$ & $\begin{array}{l}\text { Quartile } 2 \\
\geq 8.4,<8.8 \\
(\mathrm{n}=207)\end{array}$ & $\begin{array}{l}\text { Quartile } 3 \\
\geq 8.8,<9.3 \\
(\mathrm{n}=206)\end{array}$ & $\begin{array}{l}\text { Quartile } 4 \\
\geq 9.3 \\
(\mathrm{n}=203)\end{array}$ \\
\hline TyG index & $8.1 \pm 0.2$ & $8.6 \pm 0.1$ & $9.0 \pm 0.1$ & $9.8 \pm 0.5$ \\
\hline Age (years) & $63.3 \pm 13.5$ & $62.7 \pm 12.4$ & $60.6 \pm 13.5$ & $58.5 \pm 13.0^{\mathrm{ab}}$ \\
\hline Male (\%) & 54.6 & 57.5 & 59.7 & 63.1 \\
\hline Diabetes mellitus (\%) & 16.9 & 20.8 & 26.7 & $56.2^{\mathrm{abc}}$ \\
\hline Hypertension (\%) & 67.1 & 75.8 & 76.1 & 77.3 \\
\hline Coronary artery disease (\%) & 15.2 & 18.0 & 22.9 & 24.1 \\
\hline $\begin{array}{l}\text { Systolic blood pressure } \\
(\mathrm{mmHg})\end{array}$ & $133.2 \pm 21.3$ & $135.7 \pm 19.5$ & $137.8 \pm 18.4$ & $134.0 \pm 18.1$ \\
\hline $\begin{array}{l}\text { Diastolic blood pressure } \\
(\mathrm{mmHg})\end{array}$ & $75.6 \pm 11.9$ & $76.9 \pm 10.9$ & $79.0 \pm 11.1$ & $77.6 \pm 11.6$ \\
\hline $\begin{array}{l}\text { Mean arterial pressure } \\
(\mathrm{mmHg})\end{array}$ & $94.8 \pm 14.0$ & $96.5 \pm 13.0$ & $98.6 \pm 12.5$ & $96.4 \pm 12.8$ \\
\hline Body mass index $\left(\mathrm{kg} / \mathrm{m}^{2}\right)$ & $24.6 \pm 3.3$ & $25.9 \pm 3.8^{\mathrm{a}}$ & $26.6 \pm 3.6^{\mathrm{a}}$ & $27.5 \pm 3.9^{\mathrm{ab}}$ \\
\hline $\mathrm{ABI}$ & $1.12 \pm 0.13$ & $1.13 \pm 0.11$ & $1.12 \pm 0.10$ & $1.10 \pm 0.13$ \\
\hline baPWV $(\mathrm{cm} / \mathrm{s})$ & $\begin{array}{l}1717.7 \pm \\
482.5\end{array}$ & $\begin{array}{l}1746.8 \pm \\
401.9\end{array}$ & $\begin{array}{l}1804.2 \pm \\
424.5\end{array}$ & $\begin{array}{l}1739.2 \pm \\
382.0\end{array}$ \\
\hline \multicolumn{5}{|l|}{ Laboratory parameters } \\
\hline Total cholesterol (mg/dL) & $180.4 \pm 34.9$ & $188.9 \pm 41.5$ & $196.0 \pm 40.6^{\mathrm{a}}$ & $\begin{array}{l}204.5 \pm \\
51.7 \mathrm{ab}\end{array}$ \\
\hline Hematocrit (\%) & $40.4 \pm 4.8$ & $40.4 \pm 6.1$ & $41.2 \pm 5.0$ & $41.1 \pm 5.7$ \\
\hline eGFR $\left(\mathrm{mL} / \mathrm{min} / 1.73 \mathrm{~m}^{2}\right)$ & $58.7 \pm 16.8$ & $58.3 \pm 17.3$ & $58.2 \pm 20.7$ & $57.2 \pm 22.8$ \\
\hline \multicolumn{5}{|l|}{ Echocardiographic data } \\
\hline $\begin{array}{l}\text { Observed/predicted LVM } \\
(\%)\end{array}$ & $143.2 \pm 38.2$ & $147.5 \pm 47.5$ & $155.4 \pm 46.8^{\mathrm{a}}$ & $155.5 \pm 48.0^{\mathrm{a}}$ \\
\hline LA diameter (mm) & $36.3 \pm 6.3$ & $37.9 \pm 5.8$ & $38.4 \pm 6.1^{\mathrm{a}}$ & $38.3 \pm 6.0^{\mathrm{a}}$ \\
\hline LVEF (\%) & $64.8 \pm 11.9$ & $64.6 \pm 12.9$ & $63.1 \pm 13.0$ & $61.8 \pm 14.6$ \\
\hline \multicolumn{5}{|c|}{$\begin{array}{l}\text { Abbreviations. TyG index, Triglyceride-glucose index; ABI, Ankle-brachial index; } \\
\text { baPWV, brachial-ankle pulse wave velocity; eGFR, estimated glomerular filtration } \\
\text { rate; LVM, left ventricular mass; LA, left atrial; LVEF, left ventricular ejection } \\
\text { fraction. } \\
\text { a } p<0.05 \text { compared with Quartile } 1 \\
\mathrm{~b} p<0.05 \text { compared with Quartile } 2 \\
{ }^{p} p<0.05 \text { compared with Quartile } 3\end{array}$} \\
\hline
\end{tabular}


Table 2. Determinants for echocardiographic parameters using multivariable stepwise linear regression analysis

\begin{tabular}{|c|c|c|}
\hline \multirow[t]{2}{*}{ Parameters } & \multicolumn{2}{|l|}{ Multivariable (stepwise) } \\
\hline & Unstandardized coefficient $\beta$ (95\% CI) & $p$ value \\
\hline \multicolumn{3}{|c|}{ Observed/predicted LVM (per 1\%) } \\
\hline TyG index (per 1) & $4.401(-0.540,9.342)$ & 0.081 \\
\hline Age (per 1 year) & $-0.327(-0.584,-0.069)$ & 0.013 \\
\hline Diabetes mellitus & $8.957(1.349,16.566)$ & 0.021 \\
\hline Mean arterial pressure & $-0.573(-0.819,-0.326)$ & $<0.001$ \\
\hline eGFR (per $1 \mathrm{~mL} / \mathrm{min} / 1.73 \mathrm{~m}^{2}$ ) & $-0.371(-0.543,-0.199)$ & $<0.001$ \\
\hline \multicolumn{3}{|l|}{ LA diameter (per $1 \mathrm{~mm}$ ) } \\
\hline TyG index (per 1) & $0.926(0.299,1.533)$ & 0.004 \\
\hline Sex (male vs. female) & $1.931(1.039,2.822)$ & $<0.001$ \\
\hline eGFR (per $1 \mathrm{~mL} / \mathrm{min} / 1.73 \mathrm{~m}^{2}$ ) & $-0.053(-0.075,-0.030)$ & $<0.001$ \\
\hline \multicolumn{3}{|l|}{ LVEF (per 1\%) } \\
\hline TyG index (per 1) & $-2.012(-3.338,-0.686)$ & 0.003 \\
\hline Age (per 1 year) & $0.083(0.010,0.157)$ & 0.026 \\
\hline Sex ((male vs. female) & $-2.729(-4.622,-0.835)$ & 0.005 \\
\hline Hypertension & $4.931(2.807,7.056)$ & $<0.001$ \\
\hline Coronary artery disease & $-5.426(-7.789,-3.064)$ & $<0.001$ \\
\hline eGFR (per $1 \mathrm{~mL} / \mathrm{min} / 1.73 \mathrm{~m}^{2}$ ) & $0.104(0.055,-0.153)$ & $<0.001$ \\
\hline \multicolumn{3}{|c|}{$\begin{array}{l}\text { Values expressed as unstandardized coefficient } \beta \text { and } 95 \% \text { confidence interval (CI). } \\
\text { Abbreviations are the same as in Table } 1 .\end{array}$} \\
\hline
\end{tabular}

Table 3. Determinants for abnormal $A B I$, baPWV using multivariable stepwise linear regression analysis

\begin{tabular}{lll}
\hline Parameters & Multivariable (stepwise) & \\
\hline & \multicolumn{1}{l}{ Unstandardized coefficient $\beta(95 \%$} & $\begin{array}{l}p \\
\text { value }\end{array}$ \\
\hline ABI (per 0.1) & & \\
TyG index (per 1) & $-0.147(-0.279,-0.014)$ & 0.030 \\
Age (per 1 year) & $-0.014(-0.021,-0.007)$ & $<0.001$ \\
Sex (male vs. female) & $-0.215(-0.398,-0.031)$ & 0.022 \\
Coronary artery disease & $-0.291(-0.518,-0.063)$ & 0.012 \\
Total cholesterol (per 1 mg/dL) & $-0.002(-0.004,0)$ & 0.038 \\
baPWV (per 100 cm/s) & & $<0.001$ \\
$\quad$ Age (per 1 year) & $0.164(0.147,0.181)$ & $<0.001$ \\
Diabetes mellitus & $1.322(0.821,1.822)$ & $<0.001$ \\
Mean arterial pressure (per 1 & $0.162(0.144,0.179)$ & \\
mmHg) & & \\
\hline
\end{tabular}

Values expressed as unstandardized coefficient $\beta$ and $95 \%$ confidence interval (CI). Abbreviations are the same as in Table 1.

Covariates in the multivariable model included TyG index, age, gender, diabetes mellitus, hypertension, coronary artery disease, mean arterial pressure, total cholesterol, hematocrit and eGFR.

\section{Determinants of $A B I$ and baPWV in the Study Patients}

Table 3 presents the risk factors for peripheral artery disease. In multivariate stepwise linear regression analysis, a high TyG index (unstandardized coefficient $\beta,-0.147 ; 95 \% \mathrm{CI},-0.279$ to -0.014; $p=0.030)$, old age $(p<0.001)$, male sex $(p=$ $0.022)$, coronary artery disease $(p=0.012)$ and high total cholesterol $(p=0.038)$ were significantly correlated with low ABI. Regarding baPWV, old age $(p<0.001)$, diabetes $(p<0.001)$ and high mean arterial pressure $(p<0.001)$ were significantly correlated with high baPWV, but TyG index was not.

\section{Discussion}

In this study, we investigated associations between the TyG index and echocardiographic parameters of cardiac remodeling and dysfunction and indices of atherosclerosis and arterial stiffness. The results showed that a high TyG index was associated with high LAD, low LVEF and low ABI, while observed/predicted LVM and baPWV failed to show significant associations with TyG index.

The etiology of LVH includes both the impact of hemodynamic overload on the heart and other non-hemodynamic mechanisms, among which metabolic factors have been suggested to play a major role in the development of $\mathrm{LVH}$ (28). Increasing evidence has demonstrated an association between impaired glucose metabolism and LVM. In an in vivo study on insulin-treated rats, Samuelsson et al. noted myocyte hypertrophy and increased interstitial fibrosis in histochemical examinations (29). In addition, in a clinical study of 2623 subjects from the Framingham Study, Rutter et al. reported that increases in LVM and wall thickness were positively associated with worsening glucose intolerance (30). In addition, in a study of 820 elderly individuals, Sundström et al. reported that IR determined by the homeostasis model assessment formula (HOMA-IR) was related to LVM and relative wall thickness (31). In another longitudinal study of 627 participants, Cauwenberghs et al. found that hyperinsulinemia was associated with a greater increase in LVM (32). Inappropriate LVM is defined as an increase in LVM that exceeds the compensatory need of hemodynamics (33). In the present study, we found that a high TyG index was borderline significantly associated with high observed/predicted LVM. Considering that the TyG index is a recognized surrogate of IR (4), our findings are consistent with previous reports (30-32). Several mechanisms have been proposed to explain the relationship between IR and increase in LVM. High levels of insulin per se may interact with signaling pathways and genes involved in myocardial growth (29), and have adverse effects on ventricular mass through interactions between insulin, its receptor and the receptor of insulin-like growth factor 1 (IGF-1) in cardiac muscles (34). In addition, IR may decrease IGF-1 levels, which is negatively related to levels of growth hormone. Growth hormone may further contribute to an increase in LVM through its effect of proliferation and fluid retention on the heart (35). Moreover, IR may also be correlated to $\mathrm{LVH}$ through its inflammation-inducing property, as LVH has been associated with an increase in inflammatory markers (36).

Enlargement of the left atrium (LA) is considered to be the consequence of left ventricular (LV) diastolic dysfunction and subsequent LA volume or pressure overload (37). Previous studies have reported 
associations between LA enlargement and all-cause mortality (38) and future CV risk (39). In the present study, a high TyG index was associated with a large LAD. To the best of our knowledge, few studies have discussed the relationship between TyG index and LA size, while some studies have investigated the correlation between LA size and IR. In a study of Japanese hypertensive patients, Shigematsu et al. reported that IR determined by HOMA-IR was an independent predictor of echocardiographic LA enlargement (40). In addition, fasting blood glucose per se has also been associated with LA dimensions (41). Considering its relationship with LA size, LV diastolic function may help to explain the association between LA enlargement and IR. In a study of 1063 patients, Fontes-Carvalho et al. reported that HOMA-IR was independently associated with LV diastolic dysfunction, and that changes in diastolic function were present even before the onset of DM (42). In addition, Clarke et al. reported that pioglitazone, an IR-reducing medication, may improve myocardial insulin sensitivity and LV diastolic function (43). Triglycerides, another component of the TyG index, have also been correlated with LV diastolic dysfunction (44). In addition to the impact on diastolic dysfunction, inflammation and oxidative stress may also be the mechanisms underlying the association between IR and increase LA size $(45,46)$.

In the present study, we found that a high TyG index was associated with a decline in LVEF. Subclinical LV systolic dysfunction has been reported to present in patients with metabolic syndrome but no history of heart failure, myocardial infarction and $\mathrm{LVEF}<50 \%$ (47). In addition, in a large cohort study of 4425 individuals from the Cardiovascular Health Study, Banerjee et al. reported that fasting insulin levels were positively associated with the risk of heart failure (48). Moreover, in another longitudinal study, Cauwenberghs et al. found that hyperinsulinemia was related to a decrease in LVEF after follow-up for 4.7 years (32). There are some possible explanations for the association between IR and LVEF. Activation of the renin-angiotensin system (RAAS) and increased production of angiotensin II have been associated with derangement of glucose metabolism (49). In addition, interactions between IR and the RAAS may further contribute to cardiac dysfunction through different signaling transduction cascades (49) and by increasing the activity of angiotensin II in extracellular matrix deposition and cell proliferation (50). Moreover, IR and excess fatty acid have been associated with the deposition of intramyocardial lipids, and this has been suggested to result in subsequent myocardial fibrosis and systolic dysfunction (51).

Another important finding of this study is that the TyG index was negatively associated with ABI, a clinical parameter of atherosclerosis. Previous studies have demonstrated relationships between TyG index, IR and atherosclerosis. In a 1-year follow-up study of 366 patients, An et al. reported that IR was an independent predictor of atherosclerotic plaque progression in patients with coronary heart disease (52). In addition, Irace et al. reported that the TyG index was associated with carotid atherosclerosis even after adjusting for traditional risk factors of $\mathrm{CV}$ diseases (53). Several potential mechanisms may explain the associations between the TyG index, IR and atherosclerosis. Systemic factors in IR, such as hypertension, dyslipidemia and a pro-inflammatory state, may promote atherogenesis and plaque progression (54). At the cellular level, impaired insulin signaling may interfere with the normal function of vascular intimal cells that are involved in atherosclerosis, including macrophages, endothelial cells and vascular smooth muscle cells (54). In a study of 3587 Korean adults, Lee et al. reported that the TyG index was associated with increased arterial stiffness as assessed by baPWV (7). In addition, Won et al. also reported a positive association between the TyG index and arterial stiffness in a study of relatively healthy Korean subjects (55). In another recent study of 2830 elderly Chinese, Zhao et al. reported that the TyG index was associated with arterial stiffness as measured by cfPWV and baPWV, but that this was not associated with lower extremity atherosclerosis, as measured by $\mathrm{ABI}$, after adjusting for $\mathrm{CV}$ risk factors (16). However, no significant association was found between the TyG index and baPWV in the present study. In a systematic review of pulse wave velocity, only age and blood pressure were identified as being important risk factors, while other $\mathrm{CV}$ risk factors including $\mathrm{DM}$ and triglycerides only showed an insignificant contribution to cfPWV (56). This may be because the TyG index is not correlated to arterial stiffness.

To the best of our knowledge, few studies have discussed the relationships between the TyG index and echocardiographic parameters. The strength of the present study is that we explored associations between the TyG index and cardiac remodeling and dysfunction as assessed using echocardiographic parameters, including LAD and LVEF. There are also several limitations to this study. First, as all of the participants were of Chinese ethnicity, our findings may not be generalizable to other ethnicities. Second, the HOMA-IR index was not included in the analysis, however the HOMA-IR index is the gold standard method to measure IR. However, the close 
relationship between HOMA-IR and the TyG index has been demonstrated in previous studies (5). In addition, the TyG index is more cost effective and easier to calculate in routine clinical practice. Third, the study design meant that we could not draw definitive conclusions with regards to long-term clinical outcomes and causal relationships. However, we believe that our findings highlight the importance of the TyG index in patients at risk of LV dysfunction and atherosclerosis. Further prospective studies are warranted to investigate these associations.

In conclusion, in this study, we demonstrated a significant association between a high TyG index and high LAD, low LVEF and low ABI. However, the TyG index was not closely associated with baPWV. These results suggest that the TyG index, as a simple indicator of IR, may reflect cardiac remodeling and dysfunction and atherosclerosis.

\section{Acknowledgements}

The research presented in this article is supported by the grant from Kaohsiung Municipal Siaogang Hospital (grant number: kmhk-108-S-012), Kaohsiung Medical University, Kaohsiung, Taiwan.

\section{Competing Interests}

The authors have declared that no competing interest exists.

\section{References}

1. Roth GA, Johnson C, Abajobir A, Abd-Allah F, Abera SF, Abyu G, et al. Global, Regional, and National Burden of Cardiovascular Diseases for 10 Causes, 1990 to 2015. J Am Coll Cardiol. 2017; 70: 1-25.

2. Bastien M, Poirier P, Lemieux I, Despres JP. Overview of epidemiology and contribution of obesity to cardiovascular disease. Prog Cardiovasc Dis. 2014; 56: 369-81.

3. Laakso M, Kuusisto J. Insulin resistance and hyperglycaemia in cardiovascular disease development. Nat Rev Endocrinol. 2014; 10: 293-302.

4. Guerrero-Romero F, Simental-Mendia LE, Gonzalez-Ortiz M, Martinez-Abundis E, Ramos-Zavala MG, Hernandez-Gonzalez SO, et al. The product of triglycerides and glucose, a simple measure of insulin sensitivity. Comparison with the euglycemic-hyperinsulinemic clamp. J Clin Endocrinol Metab. 2010; 95: 3347-51.

5. Du T, Yuan G, Zhang M, Zhou X, Sun X, Yu X. Clinical usefulness of lipid ratios, visceral adiposity indicators, and the triglycerides and glucose index as risk markers of insulin resistance. Cardiovasc Diabetol. 2014; 13: 146.

6. Zheng R, Mao Y. Triglyceride and glucose (TyG) index as a predictor of incident hypertension: a 9-year longitudinal population-based study. Lipids Health Dis. 2017; 16: 175 .

7. Lee SB, Ahn CW, Lee BK, Kang S, Nam JS, You JH, et al. Association between triglyceride glucose index and arterial stiffness in Korean adults. Cardiovasc Diabetol. 2018; 17: 41.

8. Park K, Ahn CW, Lee SB, Kang S, Nam JS, Lee BK, et al. Elevated TyG Index Predicts Progression of Coronary Artery Calcification. Diabetes Care. 2019; 42: 1569-73.

9. Luo E, Wang D, Yan G, Qiao Y, Liu B, Hou J, et al. High triglyceride-glucose index is associated with poor prognosis in patients with acute ST-elevation myocardial infarction after percutaneous coronary intervention. Cardiovasc Diabetol. 2019; 18: 150.

10. Yan Z, Yu D, Cai Y, Shang J, Qin R, Xiao J, et al. Triglyceride Glucose Index Predicting Cardiovascular Mortality in Chinese Initiating Peritoneal Dialysis: A Cohort Study. Kidney Blood Press Res. 2019; 44: 669-78.

11. Wang L, Cong HL, Zhang JX, Hu YC, Wei A, Zhang YY, et al. Triglyceride-glucose index predicts adverse cardiovascular events in patients with diabetes and acute coronary syndrome. Cardiovasc Diabetol. 2020; 19: 80.

12. Su WY, Chen SC, Huang YT, Huang JC, Wu PY, Hsu WH, et al. Comparison of the Effects of Fasting Glucose, Hemoglobin A1c, and Triglyceride-Glucose
Index on Cardiovascular Events in Type 2 Diabetes Mellitus. Nutrients. 2019; 11.

13. Chiu H, Tsai HJ, Huang JC, Wu PY, Hsu WH, Lee MY, et al. Associations between Triglyceride-Glucose Index and Micro- and Macro-angiopathies in Type 2 Diabetes Mellitus. Nutrients. 2020; 12.

14. Fishbane S, Youn S, Kowalski EJ, Frei GL. Ankle-arm blood pressure index as a marker for atherosclerotic vascular diseases in hemodialysis patients. Am J Kidney Dis. 1995; 25: 34-9.

15. Yamashina A, Tomiyama H, Takeda K, Tsuda H, Arai T, Hirose K, et al. Validity, reproducibility, and clinical significance of noninvasive brachial-ankle pulse wave velocity measurement. Hypertension research : official journal of the Japanese Society of Hypertension. 2002; 25: 359-64.

16. Zhao S, Yu S, Chi C, Fan X, Tang J, Ji H, et al. Association between macro- and microvascular damage and the triglyceride glucose index in community-dwelling elderly individuals: the Northern Shanghai Study. Cardiovasc Diabetol. 2019; 18: 95.

17. Armstrong AC, Liu K, Lewis CE, Sidney S, Colangelo LA, Kishi S, et al. Left atrial dimension and traditional cardiovascular risk factors predict 20-year clinical cardiovascular events in young healthy adults: the CARDIA study. Eur Heart J Cardiovasc Imaging. 2014; 15: 893-9.

18. de Simone G, Verdecchia P, Pede S, Gorini M, Maggioni AP. Prognosis of inappropriate left ventricular mass in hypertension: the MAVI Study. Hypertension. 2002; 40: 470-6.

19. Su HM, Lin TH, Hsu PC, Lee CS, Lee WH, Chen SC, et al. Association of brachial-ankle pulse wave velocity, ankle-brachial index and ratio of brachial pre-ejection period to ejection time with left ventricular hypertrophy. The American journal of the medical sciences. 2014; 347: 289-94.

20. Devereux RB, Alonso DR, Lutas EM, Gottlieb GJ, Campo E, Sachs I, et al. Echocardiographic assessment of left ventricular hypertrophy: comparison to necropsy findings. The American journal of cardiology. 1986; 57: 450-8.

21. Mancia G, De Backer G, Dominiczak A, Cifkova R, Fagard R, Germano G, et al. 2007 Guidelines for the Management of Arterial Hypertension: The Task Force for the Management of Arterial Hypertension of the European Society of Hypertension (ESH) and of the European Society of Cardiology (ESC). Journal of hypertension. 2007; 25: 1105-87.

22. de Simone G, Devereux RB, Kimball TR, Mureddu GF, Roman MJ, Contaldo F, et al. Interaction between body size and cardiac workload: influence on left ventricular mass during body growth and adulthood. Hypertension. 1998; 31: 1077-82.

23. Nardi E, Palermo A, Mule G, Cusimano P, Cottone S, Cerasola G. Left ventricular hypertrophy and geometry in hypertensive patients with chronic kidney disease. Journal of hypertension. 2009; 27: 633-41.

24. Tomiyama H, Yamashina A, Arai T, Hirose K, Koji Y, Chikamori T, et al. Influences of age and gender on results of noninvasive brachial-ankle pulse wave velocity measurement--a survey of 12517 subjects. Atherosclerosis. 2003; 166: 303-9.

25. Yokoyama H, Shoji T, Kimoto E, Shinohara K, Tanaka S, Koyama H, et al. Pulse wave velocity in lower-limb arteries among diabetic patients with peripheral arterial disease. Journal of atherosclerosis and thrombosis. 2003; 10: 253-8.

26. Vickery S, Stevens PE, Dalton RN, van Lente F, Lamb EJ. Does the ID-MS traceable MDRD equation work and is it suitable for use with compensated Jaffe and enzymatic creatinine assays? Nephrology, dialysis, transplantation : official publication of the European Dialysis and Transplant Association European Renal Association. 2006; 21: 2439-45.

27. Levey AS, Bosch JP, Lewis JB, Greene T, Rogers N, Roth D. A more accurate method to estimate glomerular filtration rate from serum creatinine: a new prediction equation. Modification of Diet in Renal Disease Study Group. Annals of internal medicine. 1999; 130: 461-70.

28. de Simone G, Palmieri V, Bella JN, Celentano A, Hong Y, Oberman A, et al. Association of left ventricular hypertrophy with metabolic risk factors: the HyperGEN study. J Hypertens. 2002; 20: 323-31.

29. Samuelsson AM, Bollano E, Mobini R, Larsson BM, Omerovic E, Fu M, et al. Hyperinsulinemia: effect on cardiac mass/function, angiotensin II receptor expression, and insulin signaling pathways. Am J Physiol Heart Circ Physiol. 2006; 291: H787-96.

30. Rutter MK, Parise H, Benjamin EJ, Levy D, Larson MG, Meigs JB, et al. Impact of glucose intolerance and insulin resistance on cardiac structure and function: sex-related differences in the Framingham Heart Study. Circulation. 2003; 107: 448-54.

31. Sundstrom J, Arnlov J, Stolare K, Lind L. Blood pressure-independent relations of left ventricular geometry to the metabolic syndrome and insulin resistance: a population-based study. Heart. 2008; 94: 874-8.

32. Cauwenberghs N, Knez J, Thijs L, Haddad F, Vanassche T, Yang WY, et al. Relation of Insulin Resistance to Longitudinal Changes in Left Ventricular Structure and Function in a General Population. J Am Heart Assoc. 2018; 7.

33. Mureddu GF, Pasanisi F, Palmieri V, Celentano A, Contaldo F, de Simone G. Appropriate or inappropriate left ventricular mass in the presence or absence of prognostically adverse left ventricular hypertrophy. J Hypertens. 2001; 19: 1113-9.

34. Laustsen PG, Russell SJ, Cui L, Entingh-Pearsall A, Holzenberger M, Liao R, et al. Essential role of insulin and insulin-like growth factor 1 receptor signaling in cardiac development and function. Mol Cell Biol. 2007; 27: 1649-64. 
35. Sesti G, Sciacqua A, Scozzafava A, Vatrano M, Angotti E, Ruberto C, et al. Effects of growth hormone and insulin-like growth factor-1 on cardiac hypertrophy of hypertensive patients. J Hypertens. 2007; 25: 471-7.

36. Bo S, Mandrile C, Milanesio N, Pagani A, Gentile L, Gambino R, et al. Is left ventricular hypertrophy a low-level inflammatory state? A population-based cohort study. Nutr Metab Cardiovasc Dis. 2012; 22: 668-76.

37. Leung DY, Boyd A, Ng AA, Chi C, Thomas L. Echocardiographic evaluation of left atrial size and function: current understanding, pathophysiologic correlates, and prognostic implications. Am Heart J. 2008; 156: 1056-64.

38. Bouzas-Mosquera A, Broullon FJ, Alvarez-Garcia N, Mendez E, Peteiro J, Gandara-Sambade T, et al. Left atrial size and risk for all-cause mortality and ischemic stroke. CMAJ. 2011; 183: E657-64

39. Hoit BD. Left atrial size and function: role in prognosis. J Am Coll Cardiol. 2014; 63: 493-505.

40. Shigematsu Y, Norimatsu S, Ogimoto A, Ohtsuka T, Okayama H, Higaki J. The influence of insulin resistance and obesity on left atrial size in Japanese hypertensive patients. Hypertens Res. 2009; 32: 500-4.

41. Cuspidi C, Meani S, Fusi V, Valerio C, Catini E, Sala C, et al. Prevalence and correlates of left atrial enlargement in essential hypertension: role of ventricular geometry and the metabolic syndrome: the Evaluation of Target Organ Damage in Hypertension study. J Hypertens. 2005; 23: 875-82.

42. Fontes-Carvalho R, Ladeiras-Lopes R, Bettencourt P, Leite-Moreira A, Azevedo A. Diastolic dysfunction in the diabetic continuum: association with insulin resistance, metabolic syndrome and type 2 diabetes. Cardiovasc Diabetol. 2015; 14: 4

43. Clarke GD, Solis-Herrera C, Molina-Wilkins M, Martinez S, Merovci A, Cersosimo E, et al. Pioglitazone Improves Left Ventricular Diastolic Function in Subjects With Diabetes. Diabetes Care. 2017; 40: 1530-6.

44. Penjaskovic D, Sakac D, Dejanovic J, Zec R, Zec Petkovic N, Stojsic Milosavljevic A. Left ventricular diastolic dysfunction in patients with metabolic syndrome. Med Pregl. 2012; 65: 18-22.

45. Li JY, He Y, Ke HH, Jin Y, Jiang ZY, Zhong GQ. Plasma oxidative stress and inflammatory biomarkers are associated with the sizes of the left atrium and pulmonary vein in atrial fibrillation patients. Clin Cardiol. 2017; 40: 89-94.

46. Shoelson SE, Lee J, Goldfine AB. Inflammation and insulin resistance. J Clin Invest. 2006; 116: 1793-801.

47. Almeida AL, Teixido-Tura G, Choi EY, Opdahl A, Fernandes VR, Wu CO, et al. Metabolic syndrome, strain, and reduced myocardial function: multi-ethnic study of atherosclerosis. Arq Bras Cardiol. 2014; 102: 327-35.

48. Banerjee D, Biggs ML, Mercer L, Mukamal K, Kaplan R, Barzilay J, et al. Insulin resistance and risk of incident heart failure: Cardiovascular Health Study. Circ Heart Fail. 2013; 6: 364-70.

49. Zhou MS, Schulman IH, Zeng Q. Link between the renin-angiotensin system and insulin resistance: implications for cardiovascular disease. Vasc Med. 2012; 17: 330-41.

50. Sartori M, Ceolotto G, Papparella I, Baritono E, Ciccariello L, Calo L, et al. Effects of angiotensin II and insulin on ERK1/2 activation in fibroblasts from hypertensive patients. Am J Hypertens. 2004; 17: 604-10.

51. Lopaschuk GD, Ussher JR, Folmes CD, Jaswal JS, Stanley WC. Myocardial fatty acid metabolism in health and disease. Physiol Rev. 2010; 90: 207-58.

52. An X, Yu D, Zhang R, Zhu J, Du R, Shi Y, et al. Insulin resistance predicts progression of de novo atherosclerotic plaques in patients with coronary heart disease: a one-year follow-up study. Cardiovasc Diabetol. 2012; 11: 71.

53. Irace C, Carallo C, Scavelli FB, De Franceschi MS, Esposito T, Tripolino C, et al. Markers of insulin resistance and carotid atherosclerosis. A comparison of the homeostasis model assessment and triglyceride glucose index. Int J Clin Pract. 2013; 67: 665-72.

54. Bornfeldt KE, Tabas I. Insulin resistance, hyperglycemia, and atherosclerosis. Cell Metab. 2011; 14: 575-85.

55. Won KB, Park GM, Lee SE, Cho IJ, Kim HC, Lee BK, et al. Relationship of insulin resistance estimated by triglyceride glucose index to arterial stiffness. Lipids Health Dis. 2018; 17: 268.

56. Cecelja M, Chowienczyk P. Dissociation of aortic pulse wave velocity with risk factors for cardiovascular disease other than hypertension: a systematic review. Hypertension. 2009; 54: 1328-36. 